\title{
How Affine Arithmetic Helps Beat Uncertainties in Electrical Systems
}

\author{
Tongyu Ding, Student Member, IEEE, Riccardo Trinchero, Student Member, IEEE, Paolo \\ Manfredi, Member, IEEE, Igor S. Stievano, Senior Member, IEEE, Flavio G. Canavero, Fellow, IEEE
}

\begin{abstract}
The ever-increasing impact of uncertainties in electronic circuits and systems is requiring the development of robust design tools capable of taking this inherent variability into account. Due to the computational inefficiency of repeated design trials, there has been a growing demand for smart simulation tools that can inherently and effectively capture the results of parameter variations on the system responses. To improve product performance, improve yield and reduce design cost, it is particularly relevant for the designer to be able to estimate worstcase responses. Within this framework, the article addresses the worst-case simulation of lumped and distributed electrical circuits. The application of interval-based methods, like interval analysis, Taylor models and affine arithmetic, is discussed and compared. The article reviews in particular the application of the affine arithmetic to complex algebra and fundamental matrix operations for the numerical frequency-domain simulation. A comprehensive and unambiguous discussion appears in fact to be missing in the available literature. The affine arithmetic turns out to be accurate and more efficient than traditional solutions based on Monte Carlo analysis. A selection of relevant examples, ranging from linear lumped circuits to distributed transmissionline structures, is used to illustrate this technique.
\end{abstract}

Index Terms-Tolerance analysis, uncertainty, worst-case analysis, circuit simulation, transmission lines, affine arithmetic, interval analysis, Taylor model.

\section{INTRODUCTION}

Nowadays, there has been a growing demand for the availability of tools enabling the simulation of electrical and electronic equipment with the inclusion of the effects of system uncertainties. Circuit and device parameters suffer from various kinds of temporal and structural variations, including temperature conditions, aging and process tolerances, thus unavoidably leading to an uncertainty in the system behavior that needs to be fully characterized [1], [2].

In this framework, Monte Carlo (MC) has been used as a faithful technique which carries out repeated deterministic simulations to span the domain of parameters variation and to collect useful statistical information (e.g., see [3]). However, for large and complex systems, a single simulation can require a long time and $\mathrm{MC}$ becomes extremely inefficient due to the large number of runs required.

Recently, a set of alternative techniques aimed at overcoming the above limitation and that are based on either

T. Ding, R. Trinchero, I. Stievano and F. Canavero are with the EMC Group, Department of Electronics and Telecommunications, Politecnico di Torino, 10129 Torino, Italy (e-mail: tongyu.ding@polito.it, riccardo.trinchero@polito.it, igor.stievano@polito.it, flavio.canavero@polito.it).

P. Manfredi is with the Electromagnetics Group, Department of Information Technology, Ghent University, 9000 Gent, Belgium (e-mail: paolo.manfredi@ugent.be). probabilistic or worst-case methods have been proposed. An effective example belonging to the former class is the so called polynomial chaos (PC) theory, where random variables are expanded in terms of orthogonal polynomials [4]. This technique has been proven to be far more efficient than $\mathrm{MC}$ in the statistical simulation of circuits, cables and interconnects [2], [5], [6], [7]. The main drawback shared by both MC and PC is that the worst-case bounds arising from bounded uncertainties can be computed via post-processing and a large number of simulations only.

On the contrary, the inherent worst-case approaches directly provide an estimation of the true bounds of the responses (e.g., see [8], [9], [10]). In 1960s, Ramon E. Moore first proposed the interval analysis (IA) method [11], which represents a random parameter by means of its lower and upper bounds. The proper propagation of these bounds through a chain of calculations allows to predict the worst combination of all the uncertain parameters. Femia et al. used IA for timedomain circuit simulation in [12], where the partitioning of the parameter ranges of uncertainty is used to increase the accuracy of the IA-based computations. Although the IA is able to provide inherent worst-case results, it assumes that all random parameters are independent, without taking any possible correlation into account. As such, it in general leads to relatively large overestimation. Since MC techniques provide an underestimation of the true range, whereas IA yields an overestimation, they can be regarded as complementary tools and be applied jointly.

In order to improve the results from IA, a multivariate Taylor model (TM) approach, capable of rigorously accounting for correlation among variables, has been developed by Berz et al. [13], [14]. A TM is based on the representation of random parameters in terms of truncated Taylor expansions plus an interval remainder describing the truncation error. This method has been applied in solving ODEs, reducing the problem of overestimation in IA.

In the early 1990s, Stolfi and Comba proposed the affine arithmetic (AA) [15], which also accounts for the correlation among variables and yields much tighter and more reasonable upper and lower bounds than IA. Femia and Spagnuolo used AA to improve the circuit tolerance analysis obtained from IA [16]. Rutenbar et al. used AA for interconnect and effective capacitance modeling, and to estimate the delay distribution of variational circuits [17]. The affine technique has also been used to efficiently perform range analysis for power flow problems [18], [19] and thermal rating assessment of overhead lines [20]. However, so far, the application has been 
mainly focused on time-domain analysis of lumped circuits and systems only.

This article provides an overview of the aforementioned worst-case techniques, with a summary of their inherent features and possible limitations. Based on this discussion, it shows how AA represents a viable solution for an accurate and efficient worst-case analysis. Moreover, a self-contained description of the basic AA tool is provided, together with an overview of the necessary extensions for its effective application to the frequency-domain simulation of both lumped and distributed circuits. Specifically, the application of AA to complex- and matrix-valued calculations, which are the key building blocks allowing to operate in the frequency domain, is not available in a clear, unambiguous and systematic form in the existing literature.

The article is organized as follows. Sec. II highlights the basic features of the available state-of-the-art approaches for tolerance analysis. In Sec. III, the fundamental theory of IA, TMs and AA is briefly summarized. A comparison among these techniques is provided in Sec. IV. Sec. V introduces the partitioning technique, used to improve the accuracy of the AA predictions, along with two examples involving the frequency-domain simulation of lumped and distributed circuits. Summary and conclusions are provided in Sec. VI.

\section{STATE-OF-THE-ART OVERVIEW}

This section briefly summarizes the key features of the stateof-the-art techniques for the computation of worst-case system responses. To provide a preliminary and qualitative illustration, Fig. 1 shows the upper bound of the response of an electronic equipment affected by parameters uncertainties (solid black line) and the approximations achieved using MC, PC, IA, TM and AA. The figure provides a visualization of the intrinsic differences between the class of worst-case (IA, TM and AA) and probabilistic (MC and PC) techniques. The former methods always provide conservative bounds, i.e., an overestimation of the true worst-case response. As discussed later on, the TMand AA-based approaches are in general more precise and less conservative than IA. On the contrary, the probabilistic methods typically provide an underestimated approximation, whose accuracy increases with the number of samples and/or expansion order and can potentially get infinitely close to the true value. Hence, the probabilistic techniques achieve high precision with the sacrifice of efficiency.

A robust worst-case analysis seeks for the most pessimistic scenario. Hence, in the following, we focus on the intervalbased methods, which provide a conservative estimation of the response bounds.

\section{Worst-CASe Interval-BASEd Methods}

In this section, the essential mathematical background of the worst-case interval-based methods is outlined. IA, TM and AA are numerical techniques that replace the basic operations involved in the computation of the circuit or system response (such as sums and multiplications) and produce guaranteed enclosures for the computed quantities, taking into account any uncertainty in the input data as well as all internal truncation and round-off errors.

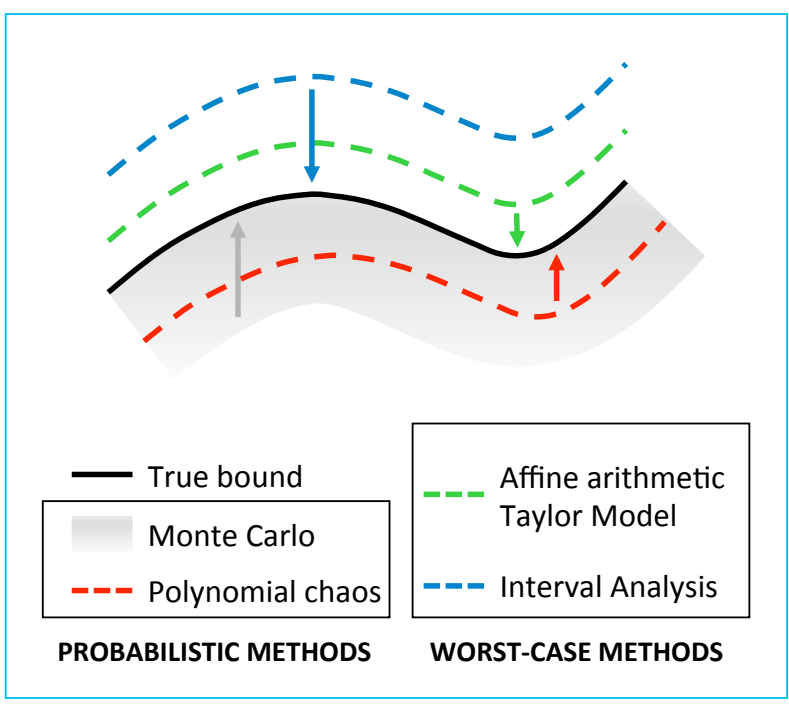

Figure 1. Approximations of the upper bound of a generic circuit or system response by means of different techniques. The solid black line is the "true" worst case, while the dashed lines are approximations obtained with PC, IA, $\mathrm{TM}$ and AA. The gray area corresponds to the superposition of MC responses, which eventually approach the true bound as the number of runs is increased.

\section{A. Interval Arithmetic}

Interval arithmetic (or interval analysis) is based on the representation of random parameters in terms of an interval bounding the possible values the parameter can assume. We will use the notation $\bar{x}=[a, b]$ to indicate that $x$ is a variable that assumes values in the interval $[a, b]$. Algebraic operations between IA-variables are carried out by computing an interval providing a guaranteed enclosure of the result. It follows that, given $\bar{x}=[a, b]$ and $\bar{y}=[c, d]$,

$$
\begin{aligned}
\bar{x}+\bar{y}= & {[a+c, b+d] } \\
\bar{x}-\bar{y}= & {[a-d, b-c] } \\
\bar{x} \times \bar{y}= & {[\min (a c, a d, b c, b d),} \\
& \max (a c, a d, b c, b d)]
\end{aligned}
$$

However, this approach is unable to track any possible correlation between the parameters and, in such a case, may lead to serious overestimation. The typical example is the calculation of $\bar{z}=\bar{x}-\bar{x}$, which according to (1) leads to $\bar{z}=[a-b, b-a] \neq[0,0]$. This trivial example shows that the application of IA without any additional modification to account for the possible correlation among variables may be too pessimistic and can be hardly used to tightly capture the worst-case bounds in a realistic chain of computations.

\section{B. Taylor Model}

The TM approach was proposed to overcome this overestimation issue. It is a "hybrid" representation of a nonlinear function of an interval variable in terms of a Taylor expansion plus an IA-remainder. Given $\bar{x}=[a, b]$,

$$
f(\bar{x})=P_{f}^{n}(x)+I_{f}
$$

where $P_{f}^{n}(x)$ is the polynomial representing the Taylor expansion of $f(x)$, truncated to a given order $n$ and usually calculated around the center of the interval, i.e., in $x_{0}=$ 
$(a+b) / 2$, whilst $I_{f}$ is an IA-variable bounding the error introduced by the truncation. Depending on the order $n, I_{f}$ can be made arbitrary small. The operations between TMs consist of a suitable combination of standard and IA calculations. Given $f(\bar{x})=P_{f}^{n}(x)+I_{f}$ and $g(\bar{x})=P_{g}^{n}(x)+I_{g}$, the calculation of $v(\bar{x})=f(\bar{x}) \pm g(\bar{x})$ is straightforward and leads to $P_{v}^{n}(x)=P_{f}^{n}(x) \pm P_{g}^{n}(x)$ (sum/difference of polynomial coefficients) and $I_{v}=I_{f} \pm I_{g}$ (IA operation). In this case, when $P_{f}^{n}(x)=P_{g}^{n}(x), P_{v}^{n}(x)=0$ and the error is limited to the (small) IA-remainder.

The product $u(\bar{x})=f(\bar{x}) \cdot g(\bar{x})$ is instead carried out by combining the standard multiplication between two polynomials, the rules of IA and the information on the bounds of the pertinent polynomial in a given domain [13], [14]. It should be noted that the order of the TM is preserved through multiplication, and the resulting IA-remainder encloses all the truncation and round-off errors in a conservative way.

The case of multiple and independent parameters is dealt with by means of multivariate Taylor expansions, whereas correlated parameters share the same variable.

It is obvious that the accuracy of the model is proportional to the order of the polynomial expansions, which increases the complexity of calculation and impacts on the efficiency. Additionally, each TM-based operation requires to correctly estimate the bounds of the IA-remainder, which can be indeed complicated in complex systems with multivariate problems.

\section{Affine Arithmetic}

Another representation of correlated interval variables is provided by the so-called AA. According to AA, the interval variables are interpreted in terms of variations around their central values, i.e., $\tilde{x}=x_{0}+x_{1} \epsilon$, with $x_{0}=(a+b) / 2$, $x_{1}=(b-a) / 2$, and where $\epsilon$ is an independent interval value ranging from -1 to +1 . Based on this alternative definition, the correlation between two variables is automatically taken into account via the same symbol $\epsilon$, leading, e.g., to the exact calculation of $\tilde{x}-\tilde{x}=x_{0}+x_{1} \epsilon-x_{0}-x_{1} \epsilon=0$.

The general definition of the affine form for a given variable with $n$ uncertainties writes:

$$
\tilde{x}=x_{0}+\sum_{i=1}^{n} x_{i} \epsilon_{i},
$$

where $x_{0}$ is the nominal or central value of the affine form, $\epsilon_{i}$ are symbolic real variables (noise symbols) whose values are unknown but assumed to lie in the interval $[-1,1]$, and $x_{i}$ are the corresponding coefficients. The AA representation is therefore similar to a first-order TM, although it encompasses all the information without resorting to additional IA terms. Based on (3) and the definition of the noise symbols, the worstcase bounds are readily given as $x_{0} \pm \sum_{i}\left|x_{i}\right|$.

Given two generic interval variables $\tilde{x}$ and $\tilde{y}$, the addition and subtraction are readily defined as $\tilde{x} \pm \tilde{y}=\left(x_{0} \pm y_{0}\right)+$ $\sum_{i=1}^{n}\left(x_{i} \pm y_{i}\right) \epsilon_{i}$. On the contrary, other operations, such as the multiplication, require special care, since the direct computation

$$
\begin{aligned}
\tilde{z} & =\tilde{x} \cdot \tilde{y} \\
& =x_{0} y_{0}+\sum_{i=1}^{n}\left(x_{0} y_{i}+y_{0} x_{i}\right) \epsilon_{i}+ \\
& +\left(\sum_{i=1}^{n} x_{i} \epsilon_{i}\right)\left(\sum_{i=1}^{n} y_{i} \epsilon_{i}\right)
\end{aligned}
$$

yields an expression that does not belong to the standard affine form as addition or subtraction do. However, the last quadratic term in (4) is suitably replaced (in a conservative way) by an additional noise symbol with a coefficient $\left.\sum_{i=1}^{n}\left|x_{i}\right| \sum_{i=1}^{n}\left|y_{i}\right|\right)$. From the above observation, it is clear that a new term is added at each multiplication, leading to a growing number of variables in a chain of computations, with a detrimental impact on the compactness of the numerical representation and on the simulation efficiency.

In order to solve this issue, Rutenbar proposed in [17] to distribute the additional quadratic terms into the existing uncertainty variables. This modification, which is used hereafter in this article, has been proven to provide a conservative approximation that bounds the true range of $\tilde{z}$ and, at the same time, successfully avoids the uncontrolled generation of new uncertainty symbols.

\section{Towards Matrix Operations}

Summarizing, both TM and AA introduce symbolic techniques into naive IA to include the correlation among variables and to help reduce the overestimation problem. For additional details on other TM- or affine-based scalar operations, such as division, exponential or square root, the reader should refer to [14] and [15], respectively.

It is important to note, however, that complex-valued $m a$ trix operations are needed to successfully apply the interval methods to the numerical solution of lumped and distributed electronic circuits in the frequency domain. Unfortunately, whereas the extension of summation and multiplication is relatively straightforward, the available literature often lacks of a systematic description of specific tools to handle other relevant matrix operations (like inversion and matrix exponential) within a given interval-based framework. In the following, we briefly review and elucidate the available information in this regard.

Complex Algebra is implemented based on the fundamental rules. Specifically, given two complex variables in rectangular form with their real and imaginary parts defined as interval values, the basic algebraic operations are readily carried out by separately accounting for the real and imaginary parts via suitable real-valued calculations.

The matrix inversion, which provides one of the key computational blocks for the frequency domain solution of a circuit, requires the joint application of interval methods along with a suitable numerical technique or algorithm. For example, consider the inversion of a matrix $\tilde{\boldsymbol{X}}$ given in affine form, and where the variability is defined by one noise symbol only:

$$
\tilde{\boldsymbol{Y}}=\tilde{\boldsymbol{X}}^{-1}=\left(\boldsymbol{X}_{0}+\boldsymbol{X}_{1} \epsilon_{1}\right)^{-1}
$$


where $\boldsymbol{X}_{0}$ and $\boldsymbol{X}_{1}$ play the same role of the scalar coefficients $x_{0}$ and $x_{1}$ in (3). Among the algorithms available in the literature, the Sherman-Morrison formula provides a clever solution for the above problem when the matrix $\boldsymbol{X}_{1}$ has unitary rank [22]. For the general case of matrices with full rank, the above procedure is still valid and is applied without modifications by splitting each matrix into the sum of rank-one matrices and carrying out the solution iteratively.

Finally, another important building block, needed for the simulation of distributed interconnects, is the matrix exponential. This operation is achieved by adopting a bilinear approximation, as outlined in [23], [24].

\section{CROSS-COMPARISON}

This section proposes a cross-comparison, where all the aforementioned worst-case techniques are applied to a simple illustrative example, in order to highlight their main features in the analysis of dynamical electric circuits.

For this purpose, the RLC circuit of Fig. 2 is considered. The circuit parameters are interval variables defined as $\bar{C}=$ $[0.4,0.6] \mathrm{F}, \bar{L}=[0.4,0.6] \mathrm{H}$, and $\bar{G}=[1.9,2.1] \mathrm{S}$, and are assumed to be all correlated.

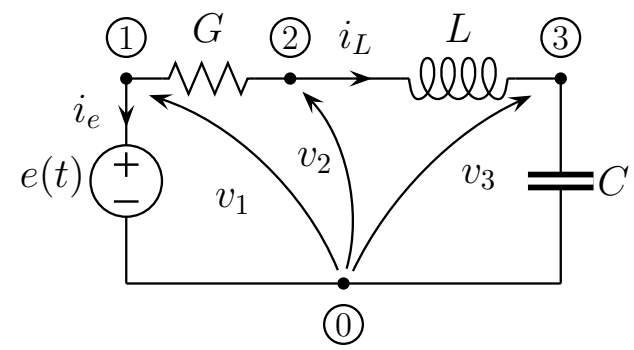

Figure 2. Illustrative RLC example used to demonstrate the performance of the various tools for the worst-case circuit analysis.

In this comparison, our analysis focuses on the magnitude (in $\mathrm{dB}$ ) of the network function $H(j \omega)=V_{3}(j \omega) / E(j \omega)$, which reads:

$$
\begin{aligned}
|H(j \omega)|_{\mathrm{dB}} & =-20 \log _{10}\left(\sqrt{\left(1-\omega^{2} L C\right)^{2}+(\omega R C)^{2}}\right) \\
& =-10 \log _{10}\left(\left(1-\omega^{2} L C\right)^{2}+(\omega R C)^{2}\right),
\end{aligned}
$$

with $R=1 / G$. It is worth noting that the above frequencydomain transfer function plays the role of a generic nonlinear function of interval-valued parameters, which needs to be processed with the IA, TM or AA tools based on their specific rules.

Fig. 3 provides the worst-case bounds of $|H(j \omega)|$ estimated via the considered techniques and compared with the spread of the response obtained with a large number of $\mathrm{MC}$ evaluations (solid green area). A zoom-in around the circuit's resonance is also displayed. This comparison shows, as expected, a large overestimation of IA, especially around the resonance. This is due to both the inherent large overestimation in treating the basic operations (1) and the inability to track the existing correlation among the variables. Furthermore, it emerges that a first-order TM significantly lacks of accuracy as well, and gives a very rough estimation. Nonetheless, a second-order TM has already a very good accuracy, far superior to IA, whereas a third-order TM is nearly indistinguishable from the MC spread. Finally, AA is also in very good agreement with the MC result, with an accuracy very close to the third-order TM.

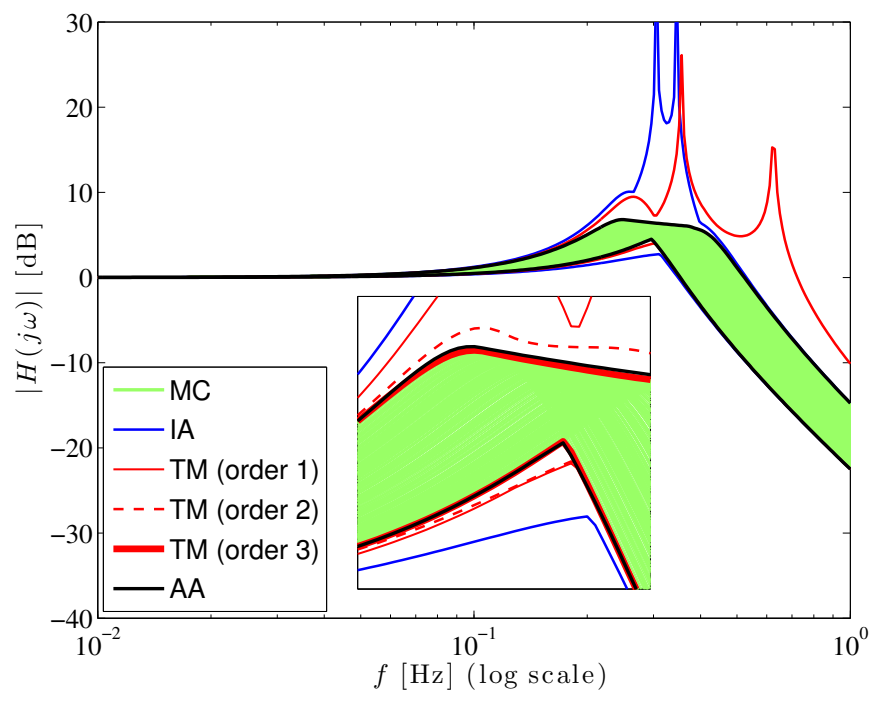

Figure 3. Bode plot (magnitude) of the transfer function $H(j \omega)=$ $V_{3}(j \omega) / E(j \omega)$ for the lumped circuit of Fig. 2. The upper and lower bounds obtained via IA (blue lines), first-, second-, and third-order TMs (red lines), and AA (black lines) are compared against the spread given by the MC samples (green area).

Summarizing, from this simple, illustrative example, it is possible to conclude that the TM requires higher-order approximations to reach good accuracy. This is likely to significantly increase the arithmetic complexity in a long chain of complex operations. On the contrary, AA turns out to be an intermediate and viable solution that allows to achieve good accuracy with a simpler mathematical representation.

In general, the response of an electrical circuit is not available in closed form, and needs to be calculated numerically. Owing to the above considerations, the AA is a good candidate to be extended in aim of an efficient frequency-domain worstcase analysis.

\section{ApPLICATIONS}

In this section, the AA is applied to the same illustrative example of Fig. 2, but in a numerical fashion, thus verifying the capability of the extended AA to deal with complexvalued matrix operations. In addition, possible enhancements currently available in the literature and aimed at improving the accuracy of the worst-case predictions are discussed. A second example involving a distributed microstrip interconnect is included as well, to highlight the feasibility of the affinebased tool to handle both lumped and distributed circuits. All the simulations are carried out using MATLAB on a Intel i74710M Quad-Core notebook operating at $2.5 \mathrm{GHz}$.

\section{A. Worst-Case Frequency-Domain Analysis}

The numerical frequency-domain solution of the circuit of Fig. 2 is obtained via the classical modified nodal analysis 
(MNA) tool [25]:

$$
\tilde{M} \tilde{W}=\tilde{J}
$$

where $\tilde{\boldsymbol{W}}=\left[V_{1}(j \omega), V_{2}(j \omega), V_{3}(j \omega), I_{L}(j \omega), I_{e}(j \omega)\right]^{T}$ collects the phasors of the unknown variables, $\tilde{\boldsymbol{J}}=$ $[0,0,0,0, E(j \omega)]^{T}$, and

$$
\begin{aligned}
\tilde{\boldsymbol{M}}= & {\left[\begin{array}{ccccc}
g_{0} & -g_{0} & 0 & 0 & 1 \\
-g_{0} & g_{0} & 0 & 1 & 0 \\
0 & 0 & j \omega C_{0} & -1 & 0 \\
0 & 1 & -1 & -j \omega L_{0} & 0 \\
1 & 0 & 0 & 0 & 0
\end{array}\right]+} \\
+ & {\left[\begin{array}{ccccc}
g_{3} \epsilon_{3} & -g_{3} \epsilon_{3} & 0 & 0 & 0 \\
-g_{3} \epsilon_{3} & g_{3} \epsilon_{3} & 0 & 0 & 0 \\
0 & 0 & j \omega C_{1} \epsilon_{1} & 0 & 0 \\
0 & 0 & 0 & -j \omega L_{2} \epsilon_{2} & 0 \\
0 & 0 & 0 & 0 & 0
\end{array}\right] . }
\end{aligned}
$$

The uncertain circuit parameters are expressed in standard affine form according to (3), with $G_{0}, C_{0}$ and $L_{0}$ corresponding to the central values of the intervals, and $G_{1}, C_{1}$ and $L_{1}$ accounting for the noise coefficients $\left(C_{0}=0.5 \mathrm{~F}, C_{1}=0.05 \mathrm{~F}\right.$, $L_{0}=0.5 \mathrm{H}, L_{1}=0.05 \mathrm{H}, G_{0}=2 \mathrm{~S}, G_{1}=0.1 \mathrm{~S}$ ).

The above matrix is interpreted as the sum of matrices in the canonical form, i.e., $\tilde{\boldsymbol{M}}=\boldsymbol{M}_{0}+\boldsymbol{M}_{1} \epsilon_{1}+\boldsymbol{M}_{2} \epsilon_{2}+\boldsymbol{M}_{3} \epsilon_{3}$, and the solution of the above problem is achieved via matrix inversion $\left(\tilde{\boldsymbol{W}}=\tilde{\boldsymbol{M}}^{-1} \tilde{\boldsymbol{J}}\right)$. This yields the response of the pertinent nodal voltages and branch currents in the complex affine form, which comprises information on the upper and lower bounds of the response.

Similarly to what was done in Sec. IV, the accuracy of the prediction via AA is assessed based on the Bode plot (magnitude) of the transfer function $H(j \omega)=V_{3}(j \omega) / E(j \omega)$, for which the same results as in Fig. 2 are found. As far as the efficiency is concerned, the CPU time required to compute the worst-case bounds for 300 frequency samples via AA is $5 \mathrm{~s}$ only, in contrast to the $32 \mathrm{~s}$ taken by $10000 \mathrm{MC}$ runs.

The good performance of the AA highlighted in the previous example is confirmed for lumped circuits that do not exhibit strong resonant behavior and with a complexity defined by a number of circuit elements in the order of 3-10. However, for more complex circuits and for a richer frequency-domain behavior, some improvements are needed and the method benefits from suitable adjustments. As an example, Fig. 4 shows the response of a lumped circuit consisting of the cascade connection of three RLC blocks like the one of Fig. 2. All the circuit parameters are in this case defined by dependent interval variables with $\pm 10 \%$ relative variation (nominal values: $C_{0}=1 \mathrm{~F}, L_{0}=1 \mathrm{H}, G_{0}=1 \mathrm{~S}$ ). The curves in the figure correspond to the Bode plot (magnitude) of the transfer function defined as the ratio between the far-end voltage response and the input voltage excitation. From this comparison, the plain AA (blue lines) only provides a rough approximation of the upper bound of the system response. However, the AA combined with the so-called uncertainty interval partitioning (UIP) [26] (black lines) leads to a major improvement, with a similar accuracy as in the previous example.

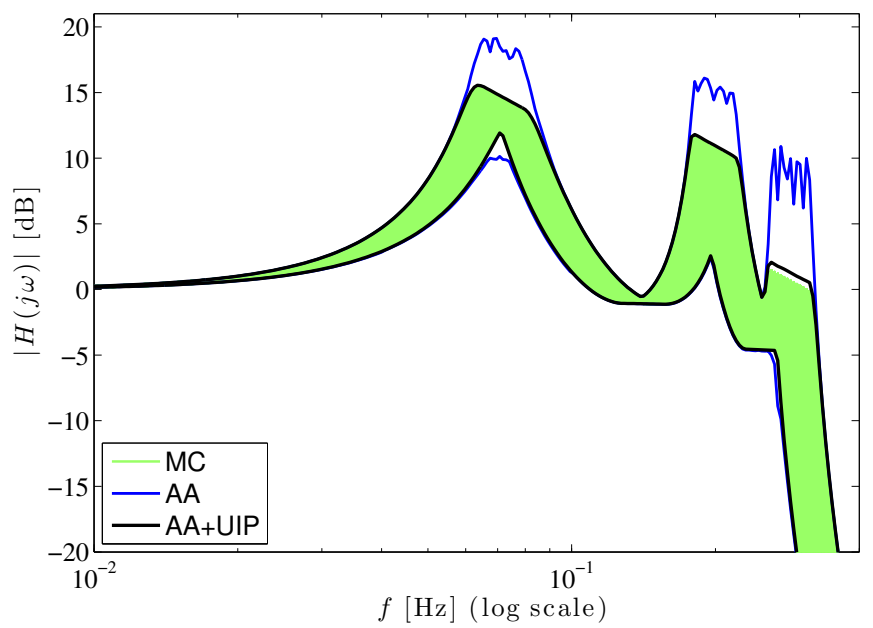

Figure 4. Frequency-domain response of the cascade connection of three lumped RLC blocks like the one of Fig. 2. Blue lines: upper and lower bounds obtained via standard AA; black lines: bounds obtained via the joint application of AA and UIP; green area: spread from MC samples.

Roughly speaking, the UIP subdivides the uncertain interval of the parameters into smaller ones and, for each subinterval, it computes the response range separately via an interval-based tool. Fig. 5 helps visualize the concept behind this technique for the case of two generic variables $x$ and $y$. This solution unavoidably impacts on the efficiency of the method which, however, in most of the applications is still more efficient than MC ( $7 \mathrm{~s}$ vs. $134 \mathrm{~s}$ for the example of Fig. 4). In the example at hand, a non-uniform partitioning is adopted, with up to 5 interval partitions in the frequency regions around the resonances.

It is relevant to remark that, in some extreme situations, a large amount of partitions with geometric progression is required to provide an accurate approximation [16]. For a detailed discussion on UIP, the interested reader is pointed to [12], [26], where the UIP is jointly applied with IA.

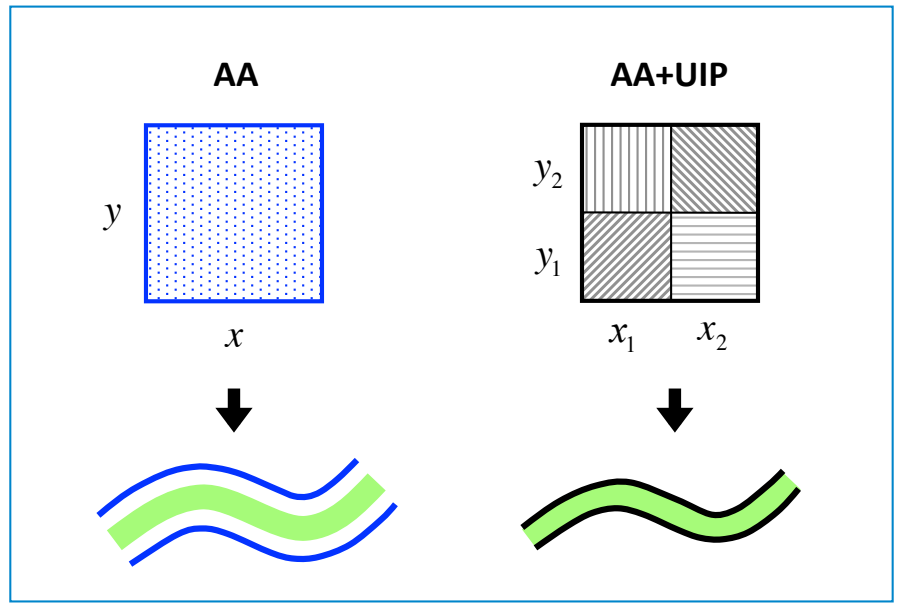

Figure 5. Graphical illustration of the UIP based on two uncertain variables (intervals $x$ and $y$ in the left panel), which are split into two subintervals each (right panel). 


\section{B. Distributed Interconnect Structures}

The second validation test case is based on the multiconductor transmission-line structure of Fig. 6, with a microstrip configuration consisting of two conductors over an ideal ground plane (top panel). For this example, the nominal values of the per-unit-length inductance and capacitance matrices $\boldsymbol{L}_{0}$ and $\boldsymbol{C}_{0}$ are calculated numerically and are

$$
\boldsymbol{L}_{0}=\left[\begin{array}{ll}
670 & 360 \\
360 & 670
\end{array}\right] \mathrm{nH} / \mathrm{m}, \quad \boldsymbol{C}_{0}=\left[\begin{array}{cc}
56 & -26 \\
-26 & 56
\end{array}\right] \mathrm{pF} / \mathrm{m}
$$

The source and load impedances (bottom panel of Fig. 6) are $R_{S}=50 \Omega$ and $R_{L}=1 \mathrm{k} \Omega$, respectively. The line length is $\ell=5 \mathrm{~cm}$.

The frequency-domain behavior of this transmission line is described via the telegrapher's equations [27]:

$$
\frac{d}{d z}\left[\begin{array}{c}
\boldsymbol{V}(z, s) \\
\boldsymbol{I}(z, s)
\end{array}\right]=-s\left[\begin{array}{cc}
0 & \boldsymbol{L} \\
\boldsymbol{C} & 0
\end{array}\right]\left[\begin{array}{c}
\boldsymbol{V}(z, s) \\
\boldsymbol{I}(z, s)
\end{array}\right]
$$

where $s=j \omega$ is the Laplace variable, whilst $\boldsymbol{V}(z, s)=$ $\left[V_{1}(z, s), V_{2}(z, s)\right]^{T}$ and $\boldsymbol{I}(z, s)=\left[I_{1}(z, s), I_{2}(z, s)\right]^{T}$ are vectors collecting the voltage and current along the two signal conductors. For the analysis, $\boldsymbol{C}$ is assumed to be an interval matrix with $\pm 10 \%$ relative variation, i.e., $\tilde{\boldsymbol{C}}=\boldsymbol{C}_{0}\left(1+0.1 \epsilon_{1}\right)$, whereas no variation is ascribed to the inductance matrix $\left(\boldsymbol{L}=\boldsymbol{L}_{0}\right)$.

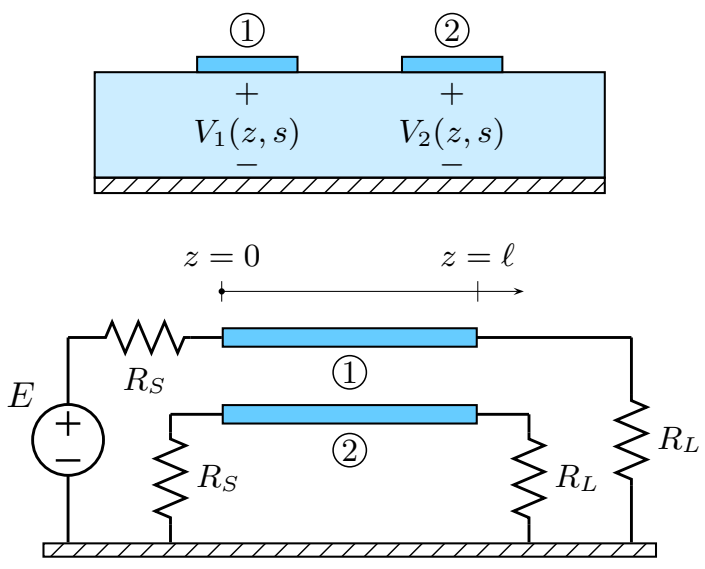

Figure 6. Three-conductor transmission-line structure. Microstrip crosssection (top panel) and circuit configuration (bottom panel).

According to [27], the fundamental step in the frequencydomain solution of (9) is the calculation of the matrix exponential

$$
\boldsymbol{\Phi}(\ell)=\exp \left(\left[\begin{array}{cc}
\mathbf{0} & -s \mathbf{L} \\
-s \mathbf{C} & \mathbf{0}
\end{array}\right] \ell\right)
$$

together with other basic operations that are carried out by means of the rules discussed in Sec. III-C and Sec. III-D.

Fig. 7 shows the spread of the magnitude of the far-end crosstalk transfer function $H(j \omega)=V_{2}(\ell, j \omega) / E(j \omega)$ up to $5 \mathrm{GHz}$, computed by means of $10000 \mathrm{MC}$ simulations (green area). The solid black lines correspond to the upper and lower bounds obtained with the AA, with the matrix exponential (10) treated as described in Sec. III-C, in conjunction with non-uniform partitioning. The accuracy and tightness of the predicted bounds confirm the potential of AA, which also provides a remarkable speed up of $10 \times$ with respect to $\mathrm{MC}$ (2 $\mathrm{min}$ vs. $20 \mathrm{~min}$ ).

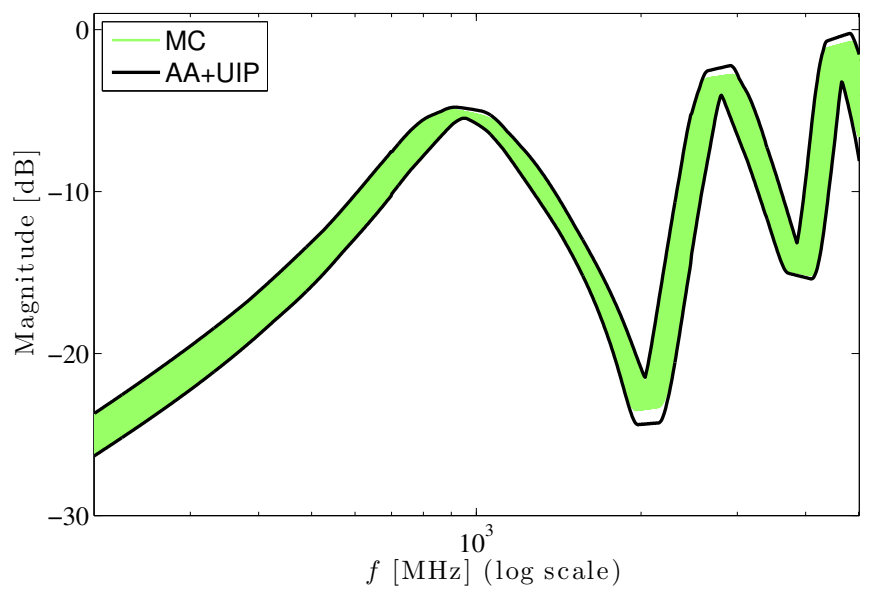

Figure 7. Bode plot (magnitude) of the far-end crosstalk transfer function for the microstrip line of Fig. 6. The number of frequency samples is 1000 . Green area: spread from MC simulations; black lines: worst-case bounds computed with AA.

\section{CONCLUSIONS}

This article provides an overview of the application of interval-based methods to the worst-case analysis of electrical circuits, with a specific focus on the frequency-domain numerical simulation via AA. Classical tools for circuit analysis are used within the affine framework, combined with UIP and accounting for complex algebra and fundamental matrix operations. The AA is successfully applied to the worstcase analysis of both lumped and distributed circuits. The performances of AA are compared in terms of both simulation time and accuracy with the traditional Monte Carlo method. Accurate results and remarkable speed-ups are achieved.

\section{ACKNOWLEDGMENT}

This work was partially supported by the Research Foundation Flanders (FWO-Vlaanderen). P. Manfredi is an FWO Postdoctoral Research Fellow.

\section{REFERENCES}

[1] A. Ajayi, P. Ingrey, P. Sewell, and C. Christopoulos, "Direct computation of statistical variations in electromagnetic problems," IEEE Trans. Electromagn. Compat., vol. 50, no. 2, pp. 325-332, May 2008.

[2] A. H. C. Smith, A. Monti, and F. Ponci, "Uncertainty and worst-case analysis in electrical measurements using polynomial chaos theory," IEEE Trans. Instrum. Meas., vol. 58, no. 1, pp. 58-67, Jan. 2009.

[3] H. Kettani and B. Barmish, "A new monte carlo circuit simulation paradigm with specific results for resistive networks," IEEE Trans. Circuits Syst. I, Reg. Papers, vol. 53, no. 6, pp. 1289-1299, Jun. 2006.

[4] D. Xiu and G. E. Karniadakis, "The Wiener-Askey polynomial chaos for stochastic differential equations," SIAM J. Sci. Comput., vol. 24, no. 2, pp. 619-644, 2002.

[5] P. Manfredi, D. Vande Ginste, D. De Zutter, and F. G. Canavero, "Stochastic modeling of nonlinear circuits via SPICE-compatible spectral equivalents," IEEE Trans. Circuits Syst. I, Reg. Papers, vol. 61, no. 7, pp. 2057-2065, Jul. 2014.

[6] I. S. Stievano, P. Manfredi, and F. G. Canavero, "Stochastic analysis of multiconductor cables and interconnects," IEEE Trans. Electromagn. Compat., vol. 53, no. 2, pp. 501-507, May 2011. 
[7] I. S. Stievano, P. Manfredi, and F. G. Canavero, "Parameters variability effects on multiconductor interconnects via Hermite Polynomial Chaos," IEEE Trans. Compon. Packag. Manuf. Technol., vol. 1, no. 8, pp. 12341239, Aug. 2011.

[8] M. Wu, D. G. Beetner, T. H. Hubing, H. Ke, and S. Sun, "Statistical prediction of reasonable worst-case crosstalk in cable bundles," IEEE Trans. Electromagn. Compat., vol. 51, no. 3, pp. 842-851, Aug. 2009.

[9] D. G. Beetner, H. Weng, M. Wu, and T. Hubing, "Validation of worstcase and statistical models for an automotive EMC expert system," in Proc. IEEE Int. Symp. Electromagn. Compat., Honolulu, HI, USA, Jul. 2007, pp. 1-5.

[10] M. Tian and C. J. R. Shi, "Worst case tolerance analysis of linear analog circuits using sensitivity bands," IEEE Trans. Circuits Syst. I, Fundam. Theory Appl., vol. 47, no. 8, pp. 1138-1145, Aug. 2000.

[11] R. E. Moore, Interval Analysis. Englewood Cliffs, NJ: Prentice-Hall, 1966.

[12] N. Femia and G. Spagnuolo, "Genetic optimization of interval arithmetic based worst case circuit tolerance analysis," IEEE Trans. Circuits Syst. I, Fundam. Theory Appl., vol. 46, no. 12, pp. 1441-1456, Dec. 1999.

[13] M. Berz and G. Hoffstätter, "Computation and application of Taylor polynomials with interval remainder bounds," Reliable Computing, vol. 4, no. 1, pp. 83-97, 1998.

[14] K. Makino and M. Berz, "Taylor models and other validated functional inclusion methods," Int. J. Pure Appl. Math., vol. 4, no. 4, pp. 379-456, 2003.

[15] J. Stolfi and L. H. de Figueiredo, "Self-validated reduced numerical methods and applications," in Monograph Brazilian Mathematics Colloquium, IMPA, Rio De Janeiro, Brazil, 1997.

[16] N. Femia and G. Spagnuolo, "True worst-case circuit tolerance analysis using genetic algorithms and affine arithmetic," IEEE Trans. Circuits Syst. I, Fundam. Theory Appl., vol. 47, no. 9, pp. 1285-1296, Sept. 2000.

[17] J. D. Ma and R. A. Rutenbar, "Fast interval-valued statistical modeling of interconnect and effective capacitance," IEEE Trans. Comput.-Aided Des. Integr. Circuits Syst., vol. 25, no. 4, pp. 710-724, Apr. 2006.

[18] A. Vaccardo, C. A. Canizares, and D. Villacci, "An affine arithmeticbased methodology for reliable power flow analysis in the presence of data uncertainty," IEEE Trans. Power Syst., vol. 25, no. 2, pp. 624-632, May 2010.

[19] J. Munoz, C. Canizares, K. Bhattacharya, and A. Vaccardo, "Affine arithmetic based methods for voltage and transient stability assessment of power systems with intermittent generation sources," in Proc. of IREP Symp., Rethymno, Greece, Aug. 2013, pp. 1-12.

[20] A. Piccolo, A. Vaccardo, and D. Villacci, "An affine arithmetic based methodology for the thermal rating assessment of overhead lines in the presence of data uncertainty," in Proc. of Power Tech Conf., Bologna, Italy, Jun. 2003.

[21] J. D. Ma and R. A. Rutenbar, "Interval-valued reduced-order statistical interconnect modeling," IEEE Trans. Comput.-Aided Des. Integr. Circuits Syst., vol. 26, no. 9, pp. 1602-1613, Sep. 2007.

[22] W. H. Press, S. A. Teukolsky, W. T. Vetterling, and B. P. Flannery, Numerical Recipes: The Art of Scientific Computing. 3rd edn., New York: Cambridge University Press, 2007.

[23] L. S. Shieh, J. Gu, and J. S. H. Tsai, "Model conversions of uncertain linear systems using the bilinear and inverse-bilinear approximation method," in Proc. of 36th Midwest Symp. Circuits Syst., Detroit, MI, USA, Aug. 1993, pp. 514-517.

[24] L. S. Shieh, X. Zou, and J. S. H. Tsai, "Model conversion of continuoustime uncertain systems via the interval geometric-series method," IEEE Trans. Circuits Syst. I, Fundam. Theory Appl., vol. 43, no. 10, pp. 851854, Oct. 1996.

[25] C.-W. Ho, A. Ruehli, and P. Brennan, "The modified nodal approach to network analysis," IEEE Trans. Circuits Syst., vol. 22, no. 6, pp. 504509, Jun. 1975.

[26] V. A. Zorich, Mathematical Analysis II. New York: Springer-Verlag Berlin Heidelberg, 2004.

[27] C. R. Paul, Analysis of Multiconductor Transmission Lines. Wiley, 1994. 